\title{
Reação de Jarisch-Herxheimer como Causa Incomum de Dor Abdominal Após o Tratamento de Sífilis: Relato de Caso
}

\section{Jarisch-Herxheimer Reaction as an Uncommon Cause of Abdominal Pain After Syphilis Treatment: Case Report}

Vânia de Oliveira ${ }^{1}$, Filipe Costa ${ }^{1}$, Cátia Neto $^{1}$, Celina Silva ${ }^{1}$, Daniel Fernandes ${ }^{1}$, Maria Tarrio ${ }^{1}$

*Autor Correspondente/Corresponding Author:

\section{RESUMO}

A sífilis é uma infeção sistémica com manifestações variáveis consoante o seu estádio clínico. Conhecida como "a grande imitadora", a sua abordagem é ainda mais desafiante quando ocorre a reação de Jarisch-Herxheimer (JH). Esta trata-se de uma reação febril aguda autolimitada que pode ocorrer nas 24 horas após tratamento de uma infeção por espiroquetas.

Reporta-se o caso de um homem de 33 anos com sífilis secundária que desenvolve uma reação de JH após três horas da administração de benzilpenicilina benzatínica 2,4 milhões de unidades internacionais, apresentando febre, agravamento do exantema inicial, dor abdominal e disfunção hepatocelular, estas últimas manifestações descritas como incomuns. Os autores discutem a fisiopatologia desta reação, as suas manifestações clínicas e recomendações de abordagem à luz da evidência atual.

PALAVRAS-CHAVE: Dor Abdominal; Efeitos Colaterais e Reações Adversas Relacionados a Medicamentos; Febre/ induzida quimicamente; Sífilis/tratamento farmacológico 


\section{ABSTRACT}

Syphilis is a systemic infection with variable manifestations depending on its clinical stage. Known as "the great imitator", its management is even more defying when the Jarisch-Herxheimer reaction occurs, which is a self-limited acute febrile reaction that occurs within 24 hours after treatment for a spirochete infection.

The authors describe the case of a 33-year-old man with a secondary syphilis who, three hours after the administration of benzathine benzylpenicillin 2.4 million international units, presents with fever, worsening of the initial cutaneous manifestations, abdominal pain and hepatocellular dysfunction, these last two described as unusual manifestations of this reaction. The authors discuss the pathophysiology of the Jarisch-Herxheimer reaction, its clinical manifestations and management recommendations according to the current evidence.

KEYWORDS: Abdominal Pain; Drug-Related Side Effects and Adverse Reactions; Fever/chemically induced; Syphilis/ drug therapy

\section{INTRODUÇÃO}

A sífilis é uma infeção crónica sistémica, maioritariamente de transmissão sexual, causada pela espiroqueta Treponema pallidum. ${ }^{1}$ Em Portugal, os dados mais recentes são referentes ao ano de 2017, no qual a taxa de incidência de casos notificados de sífilis não congénita foi de 8,8/100 000 habitantes, resultados superiores aos dados referentes ao ano de 2016 (7,5/100 000 habitantes) e 2015 (7,6/100 000 habitantes). ${ }^{2}$

Esta infeção progride por estádios, os quais apresentam manifestações clínicas consideravelmente diferentes. ${ }^{1}$ No estádio primário, ocorre geralmente a formação de uma úlcera na região genital ou perianal. No estádio secundário, múltiplas manifestações, maioritariamente cutâneas, podem surgir. Após um período de latência que pode durar várias décadas, um estádio mais avançado pode surgir, no qual a pele, coração e sistema nervoso são particularmente afetados. ${ }^{3}$ Apesar dos estádios tardios serem atualmente raros, a sífilis pode ter consequências devastadoras se não identificada e tratada. O seu tratamento varia consoante o estadio da doença. Na síflis primária, secundária ou latente precoce, a benzilpenicilina benzatínica 2,4 milhões de unidades internacionais em toma única intramuscular é o tratamento de primeira linha. No caso da sífilis latente tardia, terciária ou de duração indeterminada, preconiza-se a utilização do mesmo fármaco e dose, mas com administração semanal ao longo de três semanas consecutivas. ${ }^{4}$

Em aproximadamente 10\%-35\% dos casos de tratamento da sífilis, ocorre a reação de Jarisch-Herxheimer (JH). Trata-se de uma reação febril, aguda e autolimitada que ocorre geralmente nas primeiras 24 horas após o doente receber tratamento para uma infeção provocada por espiroquetas. ${ }^{5}$ Apesar de geralmente autolimitada, a reação de $\mathrm{JH}$ pode revelar-se confundidora de uma patologia já por si conhecida como "a grande imitadora". O caso clínico apresentado revela uma forma rara de apresentação desta reação, com dor abdominal e sinais de disfunção hepatocelular.

\section{CASO CLÍNICO}

Homem de 33 anos, com antecedentes pessoais de rinite alérgica e asma, medicado com salbutamol em SOS. Fumador (9 UMA), sem outros hábitos alcoólicos ou toxicofílicos.

Solicitou teleconsulta com o seu médico assistente por aparecimento de lesões no tronco, dorso, mãos e escroto com uma semana de evolução. Optou-se por requerer avaliação analítica (Tabela 1) e agendar consulta presencial urgente, na qual, após questionado, referia cerca de um mês antes, aparecimento de ferida no escroto à esquerda, que cicatrizou espontaneamente. Referia ainda relação sexual heterossexual desprotegida dois meses antes. Objetivamente apresentava temperatura corporal $37,3^{\circ} \mathrm{C}$, tensão arterial $117 / 75 \mathrm{mmHg}$, frequência cardíaca 72 bpm. Auscultação cardíaca e pulmonar sem alterações; abdómen inocente. Apresentava um eritema maculopapular no tronco e palmas das mãos, não pruriginoso. No escroto, à esquerda, apresentava uma mácula dura ao toque, indolor. Eram palpáveis adenopatias inguinais moles, móveis e indolores.

Foi assumida sífilis secundária, pelo que foi feita notificação SINAVE e administrada toma única de benzilpenicilina benzatínica 2,4 milhões de unidades internacionais/6,5 mL. Cerca de três horas depois, recorreu novamente a consulta presencial urgente por dor no quadrante superior direito do abdómen. Ao exame objetivo apresentava-se febril (temperatura timpânica $38,5^{\circ} \mathrm{C}$ ), normotenso e normocárdico. $\mathrm{O}$ abdómen apresentava-se doloroso à palpação do hipocôndrio 
TABELA 1. Avaliação analítica inicial realizada ao doente.

\begin{tabular}{|l|c|}
\hline Parâmetro & Valor \\
\hline Hemoglobina $(\mathrm{N}=13-18 \mathrm{~g} / \mathrm{dL})$ & $14,7 \mathrm{~g} / \mathrm{dL}$ \\
\hline Leucócitos $\left(\mathrm{N}=4-11 \times 10^{3} / \mu \mathrm{L}\right)$ & $8,13 \times 10^{3} / \mu \mathrm{L}$ \\
\hline Neutrófilos $\left(\mathrm{N}=4-11 \times 10^{3} / \mu \mathrm{L}\right)$ & $7,5 \times 10^{3} / \mu \mathrm{L}(59,7 \%)$ \\
\hline Plaquetas $\left(\mathrm{N}=4-11 \times 10^{3} / \mu \mathrm{L}\right)$ & $342 \times 10^{3} / \mu \mathrm{L}$ \\
\hline VDRL & Positivo $(1 / 128)$ \\
\hline TPHA & Reativo $(1 / 2560)$ \\
\hline Anticorpos HIV 1 e 2 & Negativos \\
\hline Anticorpo anti-HCV & Negativo \\
\hline Antigénio HBs & Negativo \\
\hline Anticorpo anti-HBc & Negativo \\
\hline Anticorpo anti-HBe & Negativo \\
\hline Anticorpo anti-HBs & Positivo \\
\hline
\end{tabular}

TABELA 2. Avaliação analítica no Serviço de Urgência.

\begin{tabular}{|l|c|}
\hline Parâmetro avaliado & Valor OBTIDO \\
\hline Hemoglobina $(\mathrm{N}=13-18 \mathrm{~g} / \mathrm{dL})$ & $14,4 \mathrm{~g} / \mathrm{dL}$ \\
\hline Leucócitos $\left(\mathrm{N}=4-11 \times 10^{3} / \mathrm{\mu L}\right)$ & $11,7 \times 10^{3} / \mu \mathrm{L}$ \\
\hline Neutrófilos $(\mathrm{N}=1,600-8,000 / \mu \mathrm{L})$ & $10,1 \times 10^{3} / \mu \mathrm{L}(86,7 \%)$ \\
\hline Plaquetas $\left(\mathrm{N}=150-450 \times 10^{3} / \mathrm{\mu L}\right)$ & $345 \times 10^{3} / \mathrm{\mu L}$ \\
\hline Bilirrubina total $(\mathrm{N}<1,2 \mathrm{mg} / \mathrm{dL})$ & $0,88 \mathrm{mg} / \mathrm{dL}$ \\
\hline Bilirrubina direta $(\mathrm{N}<0,3 \mathrm{mg} / \mathrm{dL})$ & $0,22 \mathrm{mg} / \mathrm{dL}$ \\
\hline AST/TGO $(\mathrm{N}=15-37 \mathrm{UI} / \mathrm{L})$ & $252 \mathrm{UI} / \mathrm{L}$ \\
\hline ALT/TGP $(\mathrm{N}=30-65 \mathrm{UI} / \mathrm{L})$ & $156 \mathrm{UI} / \mathrm{L}$ \\
\hline GGT $(\mathrm{N}=5-85 \mathrm{UI} / \mathrm{L})$ & $768 \mathrm{UI} / \mathrm{L}$ \\
\hline FA $(\mathrm{N}=45-117 \mathrm{UI} / \mathrm{L})$ & $229 \mathrm{UI} / \mathrm{L}$ \\
\hline LDH $(\mathrm{N}=84-246 \mathrm{UI} / \mathrm{L})$ & $364 \mathrm{UI} / \mathrm{L}$ \\
\hline PCR $(\mathrm{N}<3 \mathrm{mg} / \mathrm{L})$ & $59,7 \mathrm{mg} / \mathrm{dL}$ \\
\hline Pesquisa SARS-CoV-2 & Negativo
\end{tabular}

TABELA 3. Avaliação analítica seis meses após o tratamento da sífilis.

\begin{tabular}{l|c}
\hline Parâmetro avaliado & Valor OBTIDO \\
\hline Hemoglobina $(\mathrm{N}=13-18 \mathrm{~g} / \mathrm{dL})$ & $15,7 \mathrm{~g} / \mathrm{dL}$ \\
\hline Leucócitos $\left(\mathrm{N}=4-11 \times 10^{3} / \mu \mathrm{L}\right)$ & $10,31 \times 10^{3} / \mathrm{\mu L}$ \\
\hline Neutrófilos $(\mathrm{N}=1,600-8,000 / \mu \mathrm{L})$ & $5,22 \times 10^{3} / \mu \mathrm{L}(86,7 \%)$ \\
\hline Plaquetas $\left(\mathrm{N}=150-450 \times 10^{3} / \mu \mathrm{L}\right)$ & $361 \times 10^{3} / \mu \mathrm{L}$ \\
\hline Bilirrubina total $(\mathrm{N}<1,2 \mathrm{mg} / \mathrm{dL})$ & $0,4 \mathrm{mg} / \mathrm{dL}$ \\
\hline Bilirrubina direta $(\mathrm{N}<0,3 \mathrm{mg} / \mathrm{dL})$ & $0,2 \mathrm{mg} / \mathrm{dL}$ \\
\hline AST/TGO $(\mathrm{N}=15-37 \mathrm{UI} / \mathrm{L})$ & $25 \mathrm{UI} / \mathrm{L}$ \\
\hline ALT/TGP $(\mathrm{N}=30-65 \mathrm{UI} / \mathrm{L})$ & $48 \mathrm{UI} / \mathrm{L}$ \\
\hline GGT $(\mathrm{N}=5-85 \mathrm{UI} / \mathrm{L})$ & $52 \mathrm{UI} / \mathrm{L}$ \\
\hline FA (N=45-117 UI/L) & $94 \mathrm{UI} / \mathrm{L}$ \\
\hline LDH (N= $84-246 \mathrm{UI} / \mathrm{L})$ & $174 \mathrm{UI} / \mathrm{L}$ \\
Albumina $(\mathrm{N}=3,5-5,2 \mathrm{~g} / \mathrm{dL})$ & $5,0 \mathrm{~g} / \mathrm{dL}$ \\
VDRL & Positivo (1/1)
\end{tabular}

direito. Era ainda evidente um agravamento do eritema prévio. Foi referenciado ao Serviço de Urgência, onde realizou estudo complementar com eletrocardiograma, radiografia do tórax e abdómen e ecografia abdominal, os quais não revelaram alterações. A Tabela 2 sumariza os resultados analíticos obtidos, dos quais se destacava discreta leucocitose com neutrofilia e disfunção hepatocelular com elevação da TGO, TGP e GGT cerca de três vezes o valor de referência. Realizou ainda teste SARS-CoV-2, que se revelou negativo.

O doente foi medicado com metamizol magnésico, com alívio da dor. Teve alta do Serviço de Urgência, referenciado para a consulta externa de Medicina Interna. Após dois dias, foi feita reavaliação telefónica pelo médico assistente, apresentando-se o doente melhorado clinicamente. Solicitado estudo analítico de reavaliação da função hepática, que o doente apenas realizou após seis meses e que se encontra sumarizado na Tabela 3. Verificou-se uma normalização da função hepática, bem como uma resposta adequada ao tratamento.

\section{DISCUSSÃO}

A reação de JH é uma reação inflamatória transitória que surge tipicamente em até 24 horas após o tratamento de uma infeção por espiroquetas. ${ }^{6}$ Apesar do mecanismo fisiopatológico por detrás da reação de $\mathrm{JH}$ não estar completamente estabelecido, pensa-se que resulte da fagocitose acelerada por parte dos leucócitos polimorfonucleares, seguida de libertação de lipoproteínas, citocinas e complexos imunes de microrganismos mortos. $^{5-7}$ Consistentemente, no caso da sífilis, esta reação é mais frequentemente observada em doentes com títulos superiores nos testes não-treponémicos. ${ }^{6}$ É, portanto, mais frequente em casos de sífilis primária e secundária, sendo rara na sífilis latente e muito rara na sífilis tardia. ${ }^{8}$

Apesar de raramente associada a morbilidade a longo prazo, esta reação pode ser sintomaticamente grave, manifestando-se sob a forma de sintomas constitucionais (febre, tremores, cefaleia, mialgias) e agravamento do eritema inicial. $6,8,9$ Pela sua apresentação, é frequentemente confundida com uma alergia medicamentosa. ${ }^{9}$ Manifestações menos comuns incluem meningite, insuficiência respiratória, renal ou hepática, alterações do estado de consciência, síndrome coronária aguda, acidente vascular cerebral e contrações uterinas na gravidez, podendo estar associada à indução de trabalho de parto. ${ }^{6,10}$

Apesar da sua apresentação súbita e inespecífica, é geralmente transitória, apresentando reversão espontânea em até 24 horas. Não é conhecida qualquer forma de prevenção do seu aparecimento, sendo que paracetamol ou anti-histamínicos prévios à administração de penicilina não mostraram prevenir esta reação. ${ }^{6}$ Foram 
também utilizados corticoesteroides, contudo, não é claro que o seu efeito seja suficientemente benéfico para que sejam recomendados. ${ }^{10}$ Anti-inflamatórios não esteroides e outros antipiréticos podem ser usados para alívio sintomático, no sentido de reduzir a gravidade dos sintomas e duração da reação. ${ }^{8}$

Diferentes antibióticos usados em infeções por espiroquetas encontram-se em estudo como alternativas no tratamento da sífilis para reduzir a incidência da reação de $\mathrm{JH}$, não existindo ainda evidência do seu benefício. Estratégias preventivas com agentes mais recentes tais como anticorpos anti-TNFa parecem promissoras, contudo a sua utilidade não foi ainda comprovada. ${ }^{8}$

Não podendo ser prevenida, a reação de JH pode, contudo, ser antecipada, pelo que os doentes devem ser informados dos possíveis sinais e sintomas e aconseIhados a contactar o seu médico assistente caso uma reação grave ocorra. ${ }^{8}$ Paralelamente, para o clínico que não se encontre alerta para esta possível reação, o diagnóstico diferencial pode revelar-se complexo e a investigação adicional infrutífera, existindo possibilidade de diagnósticos erróneos, nomeadamente de alergia a penicilina. $\bigcirc$ presente trabalho tem como objetivo alertar para esta reação e sua abordagem, por forma a obviar erros de diagnóstico ou terapêutica e para que seja feito a correta abordagem destas situações.

\section{APRESENTAÇÕES/ PRESENTATIONS}

Este trabalho foi apresentado sob a forma de comunicação oral no $27^{\circ}$ Encontro do Internato de Medicina Geral e Familiar da Zona Norte.

\section{RESPONSABILIDADESÉTICAS}

CONFLITOS DE INTERESSE: Os autores declaram a inexistência de conflitos de interesse na realização do presente trabalho.

FONTES DE FINANCIAMENTO: Não existiram fontes externas de financiamento para a realização deste artigo.

CONFIDENCIALIDADE DOS DADOS: Os autores declaram ter seguido os protocolos da sua instituição acerca da publicação dos dados de doentes.

CONSENTIMENTO: Consentimento do doente para publicação obtido.

PROVENIÊNCIA E REVISÃO POR PARES: Não comissionado; revisão externa por pares.

\section{ETHICAL DISCLOSURES}

CONFLICTS OF INTEREST: The authors have no conflicts of interest to declare.

FINANCING SUPPORT: This work has not received any contribution, grant or scholarship.

CONFIDENTIALITY OF DATA: The authors declare that they have followed the protocols of their work center on the publication of data from patients.

PATIENT CONSENT: Consent for publication was obtained.

PROVENANCE AND PEER REVIEW: Not commissioned; externally peer reviewed.

\section{REFERÊNCIAS}

1. Forrestel AK, Kovarik CL, Katz KA. Sexually acquired syphilis: Historical aspects, microbiology, epidemiology, and clinical manifestations. J Am Acad Dermatol. 2020:82:1-14. doi: 10.1016/j.jaad.2019.02.073

2. Instituto Nacional de Estatística. Portal do INE Portal [Internet]. Taxa de incidência de casos notificados de doenças de declaração obrigatória por 100000 habitantes (Série 2015 - N.`) por Local de residência, Grupo etário e Doenças de declaração obrigatória; Anual - DGS, Casos Notificados de Doenças de Declaração Obrigatória. 2021 [cited 2021 Mar 7]. Available from: https://www.ine.pt/xportal/xmain?xpid=INE\&xpgid=ine indicadores\&indOcorrCod $=0009142 \&$ contexto=bd\&sel$\mathrm{Tab}=\operatorname{tab} 2 \& \mathrm{xlang}=\mathrm{pt}$.

3. Stock I. Syphilis - an update. Med Monatsschr Pharm. 2017; 40:113-9.

4. WHO Department of Reproductive Health and Research. WHO Guidelines for the Treatment of Treponema pallidum (syphilis). Geneva: WHO; 2016.

5. Yang C, Lee N, Lin Y, Lee H, Ko W, Liao C, et al. Jarisch-Herxheimer Reaction after Penicillin Therapy among Patients with Syphilis in the Era of the HIV Infection Epidemic: Incidence and Risk Factors. Clin Infect Dis. 2010;51:976-9. doi: 10.1086/656419.

6. Forrestel AK, Kovarik CL, Katz KA. Sexually acquired syphilis: Laboratory diagnosis, management, and prevention. J Am Acad Dermatol. 2020;82:17-28. doi: 10.1016/j.jaad.2019.02.074.

7. Pound MW, May DB. Proposed mechanisms and preventative options of Jarisch-Herxheimer reactions. J Clin Pharm Ther. 2005;30:291-5. doi: 10.1111/j.1365-2710.2005.00631.x.

8. Belum GR, Belum VR, Chaitanya Arudra SK, Reddy BS. The Jarisch-Herxheimer reaction: Revisited. Travel Med Infect Dis. 2013;11:231-7. doi: 10.1016/j.tmaid.2013.04.001.

9. Miller WM, Gorini F, Botelho G, Moreira C, Barbosa AP, Pinto AR, et al. Jarisch-Herxheimer reaction among syphilis patients in Rio de Janeiro, Brazil. Int J STD AIDS. 2010;21:806-9.

10. Butler T. The Jarisch-Herxheimer Reaction After Antibiotic Treatment of Spirochetal Infections: A Review of Recent Cases and Our Understanding of Pathogenesis. Am J Trop Med Hyg. 2017;96:46-52. doi: 10.4269/ajtmh.16-0434 